\title{
Panfacial Bone Fracture and Medial to Lateral Approach
}

Jiye Kim,

Jin-Hee Choi, Yoon Kyu Chung, Sug Won Kim

Department of Plastic and Reconstructive Surgery, Yonsei University Wonju College of Medicine, Wonju, Korea

No potential conflict of interest relevant to this article was reported.
Panfacial bone fracture is challenging. Even experienced surgeons find restoration of original facial architecture difficult because of the severe degree of fragmentation and loss of reference segments that could guide the start of facial reconstruction. To restore the facial contour, surgeons usually follow a general sequence for panfacial bone reduction. Among the sequences, the bottom-to-top and outside-in sequence is reported to be the most widely used in recent publications. However, a single sequence cannot be applied to all cases of panfacial fractures because of the variations in panfacial bone fracture patterns. In this article, we intend to find the reference and discuss the efficacy of inside-out sequence in facial bone fracture reconstruction.

Keywords: Panfacial / Base sequence

\section{INTRODUCTION}

Panfacial bone fractures are defined as facial fractures simultaneously involving the upper, middle, and lower thirds of the face. Fractures of the frontal bone, maxilla, zygomatic complex, nasoethmoid-orbital (NEO) region, and mandible are the most common [1-3]. In simpler terms, panfacial bone fractures involve the midface and mandible [4]. Even experienced surgeons find restoration of the original facial architecture difficult because of the severe degree of fragmentation and the loss of all reference segments that could guide the start of facial reconstruction $[4,5]$. Panfacial bone fractures are often accompanied by soft tissue trauma and destruction of the bony framework, which may result in malocclusion or facial deformities, including "dish" face deformity, loss of facial height or projection, increased facial width, and enophthalmos [6]. Herein, we review the efficacy of the inside-out sequence for the reduction of panfacial bone fractures.

\section{Correspondence: Jiye Kim}

Department of Plastic and Reconstructive Surgery, Wonju Severance Christian Hospital, Yonsei University Wonju College of Medicine, 20 Ilsan-ro, Wonju 26426, Korea

E-mail: gen80@yonsei.ac.kr

Received April 18, 2016 / Revised June 25, 2016 / Accepted June 25, 2016

\section{EPIDEMIOLOGY OF PANFACIAL BONE FRACTURE}

Panfacial fractures are usually caused by high-energy injuries (e.g., motor vehicle or gunshot injuries) [7]. Panfacial fractures account for $4 \%-10 \%$ of all facial fractures. In Korea, the incidence was reported to account for $6.59 \%$ of all facial bone fractures [8]. The most common site of mandibular fracture is the symphysis (33.5\%), followed by the condyle (31.1\%) and body (17.1\%) [9].

\section{HISTORY OF THE MANAGEMENT OF PANFACIAL FRACTURES}

In the 1980s and early 1990s, craniofacial surgeons established the principles of wide exposure and direct visualization of fracture alignment for accurate craniofacial bone reduction. When these principles were applied, the sequence of alignment restoration was influenced [10]. The concept of facial buttresses was emphasized as the key to restoration of skeletal framework, and panfacial reconstructions began with the reduction of the frontal bone and proceeded with the midfacial bone alignment. Using the recon- 
structed maxillary framework as a template, the lower face was reconstructed last (top-to-bottom sequence) $[11,12]$.

Following the advent of rigid internal fixation, surgeons started reduction of facial fractures with the mandibular condyle [13]. The condyles determine the facial posterior height, and restoration of this height allows the mandible, which is the strongest bone of the facial skeleton, to be used as a template for panfacial bone reduction [14]. Because of this, the bottom-to-top sequence is widely used in craniofacial surgery today.

\section{VARIOUS SEQUENCES OF PANFACIAL BONE REDUCTION}

As no clear classification of panfacial bone fractures is available, various sequences of reduction (bottom-to-top, top-to-bottom, inside-out, and outside-in) are used in combination to restore facial contour. Numerous studies have compared combinations of these reduction sequences. However, the efficacy of inside-out or outside-in sequences have not been assessed independently of bottom-to-top or top-to-bottom sequences [4-7,9]. The "bottomto-top and outside-in" approach is the most widely used method in the panfacial bone reduction [2,4,5,7,9]. Gruss and Phillips [11] advised starting panfacial reconstructions with reduction of the zygomatic arch and malar projection to establish the outer facial frame and to provide upper facial width and projection before NEO, maxillary, and mandibular reconstruction (Fig. 1). Merville [15] suggest the frontozygomatic suture line should be reduced first in panfacial bone fractures because this important structure determines facial width and projection. As NEO fracture fragments are fragile, it is difficult to find a stable fixation point in naso-ethmoid-orbital area. Therefore, experts often recommend the outside-in sequence for reconstruction of panfacial bone fractures.

\section{INSIDE-OUT SEQUENCE}

Few authors have recommend the inside-out sequence in favor of the outside-in sequence. For panfacial fractures involving the
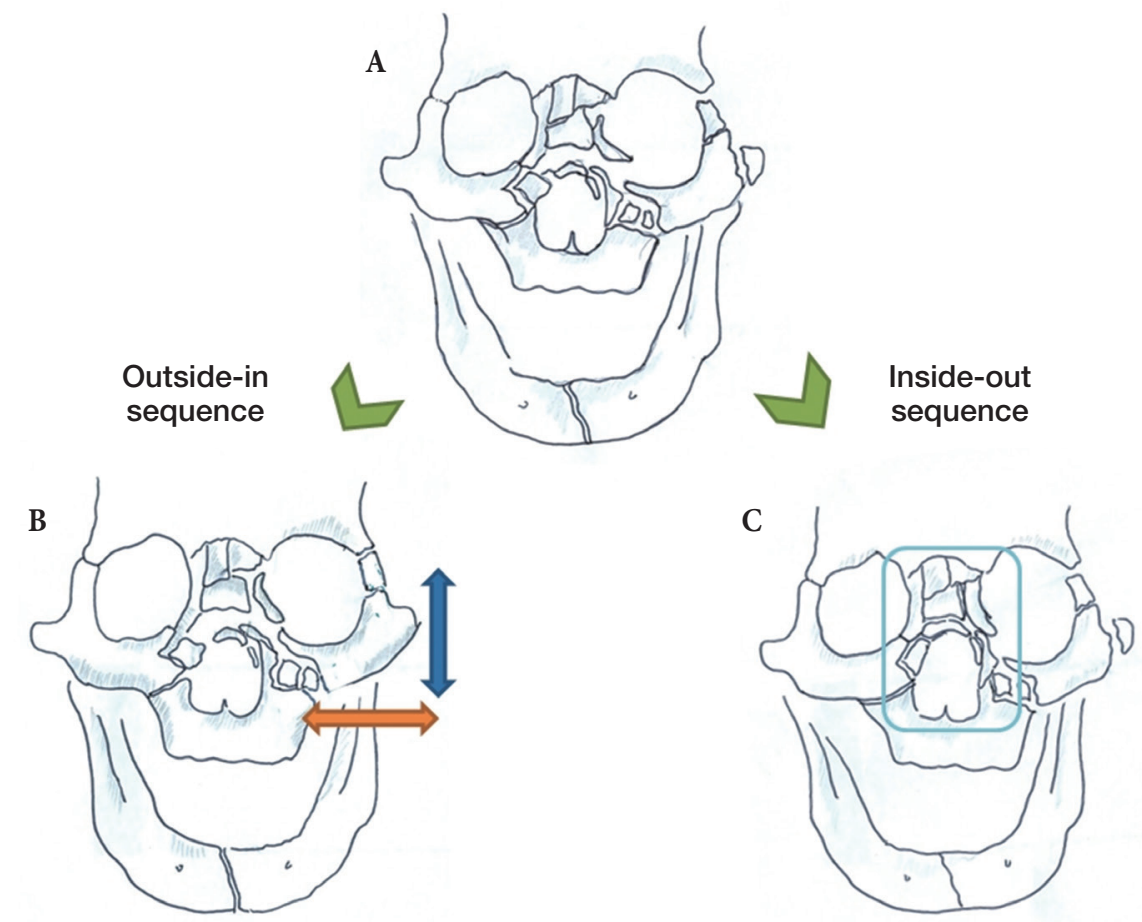

Fig. 1. (A) An example of a panfacial bone fracture. (B) Most studies have advised starting facial bone reconstruction with the reduction of zygomatic arch and malar projection to establish the outer facial frame and to provide upper facial width and projection before nasoethmoid-orbital (NEO) region. (C) As the NEO fracture fragments are fragile, it is difficult to find a stable fixation point in this area. 
NEO, Merville [15] suggests initially managing the NEO fracture $[4,5]$. However, he also emphasizes the importance of the outer facial frame and that the NEO fracture should be treated within the fronto-maxillary and zygomatic frame.

In cases of bilateral condylar fractures, Pau et al. [14] suggest inverting the order of repair from the outside-in sequence (Fig. 2). They suggest starting with the mandibular symphysis for several reasons. First, internal fixation and reduction in the condylar area are challenging owing to the lack of exposure. Only a single fourhole plate is most commonly used to fixate the condyle neck because of the small amount of bone and lack of exposure in the condyle neck area. Single fixation of condyle fractures are may become unstable during symphysis correction. Second, symphysis could be fixed more stable than condyle, and with two-point rigid fixation of symphysis, condyle could be stably corrected following symphysis.

In the absence of bilateral condylar fractures, surgeons generally find the outside-in sequence to be the most reliable sequence
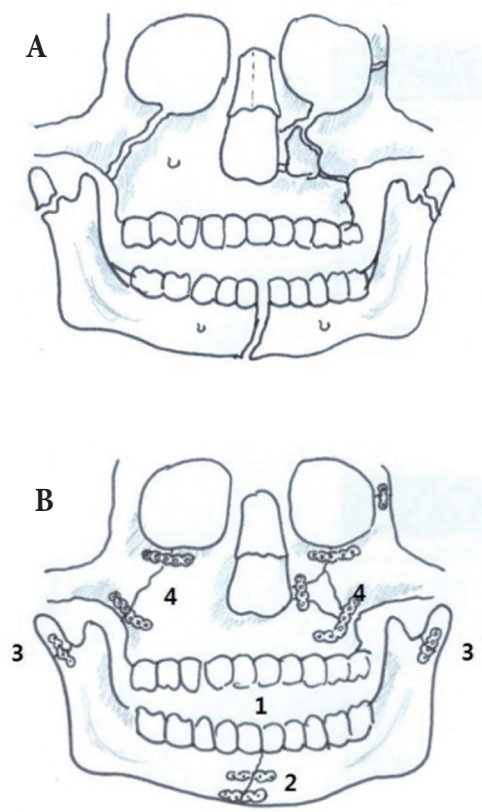

Fig. 2. The sequence of reduction and fixation in special condition like the panfacial bone fracture involves the bilateral condyle fractures. (A) Preoperative three-dimensional computed tomography imaging of a patient with a panfacial bone fracture. (B) The sequence of panfacial bone management was occlusion plane, symphysis/parasymphysis, bilateral condyle, and zygomaticomaxillary fracture. for panfacial bone correction because malar projection and condylar height are the most important point to decide the facial contour. Medial fractures such as NEO or symphysis, parasymphysis fractures should be followed according to frame that is decided by projection and height.

\section{COMPARISON OF APPROACH METHOD}

Degala et al. [4] have compared the bottom-to-top and inside-out sequences (5 patients) with the top-to-bottom and outside-in sequences (6 patients). In both groups, the patients attained good occlusion, and no statistically significant differences were found for mouth opening between the groups. Facial asymmetry, which was observed in two patients of each group, showed no significant difference in the final treatment outcome. As both sequences yield similar clinical outcomes, the decision of which sequence to use depends on the fracture pattern and surgeon preference.

\section{OUR EXPERIENCE BASED SUGGESTIONS}

We reviewed a total of 53 patients who received open reduction at a single academic institution (Wonju Severance Christian Hospital) between January 2011 and December 2015. Most of these fractures were suitable for outside-in approach. Given that the malar projection is the most important part in panfacial bone reduction, we prefer the outside-in sequence over the inside-out sequence. We have not experienced a complicated case like Pau's report [14], which is presented with fractures of the mandibular symphysis combined with bilateral extracapsular condylar fractures. On the contrary to most cases, we prefer the inside-out approach in special cases that have frontal bone fracture near the nasofrontal junction. In cases without comminuted fracture in nasoethmoid area but open wounds near the frontal bone fracture site, we start the reduction from the center of frontal bone through the laceration and with rigid fixation of fracture segments to frontal bone that would be a reliable landmark, zygomaticomaxillary segments were reduced (Fig. 3). We believe that the frontal bone is strong enough to provide the supported needed 

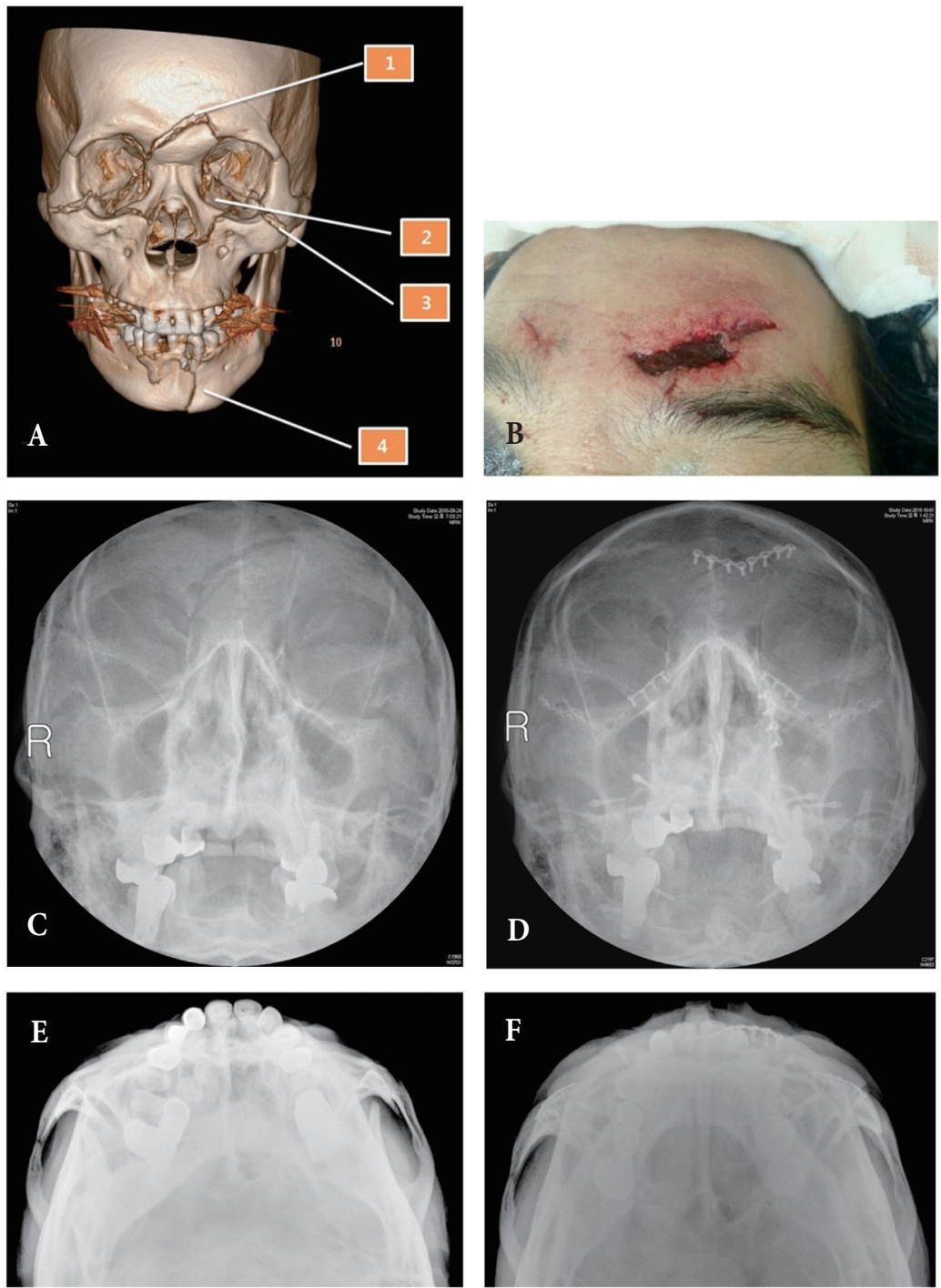

Fig. 3. This 61-year-old male was injured by bicycle accident. Frontal bone, bilateral naso-maxillary and zygomaticomaxillary fracture and mandible parasymphysis fracture was observed. (A, B) Our fracture reduction sequence. There was no severe naso-ethmoid-orbital area fracture, and the midface fracture did not affect the occlusion. We started the segment reduction from the frontal bone through the laceration wound. (C, E) Water's view and zygomatic arch view prior to operation. (D, F) Water's view and zygomatic arch view at 3 weeks after operation.

for reducing the nasomaxillary and supraorbital rim buttresses.

However, if comminuted fracture exist in naso-ethmoid-orbital area, stabilization of frontal bone near the nasofrontal junction area cannot be continued to stabilization of nasomaxillary buttress. Medial frontal bone could be a suitable starting point to manage the multiple fracture segments when comminuted nasoethmoid-orbital bone fracture was not combined.

\section{CONCLUSION}

Independently measuring the reliability and efficacy of the insideout sequence is challenging because the sequence alone is not sufficient in treating panfacial bone fractures. As a result, this sequence is often combined with either the bottom-to-top sequence or top-to-bottom sequence. Between the inside-out approach and outside-in, most craniofacial surgeons recommend the outside-in sequence because the approach has a great advantage in restoring 
the outer facial frame and projection. However, a consistent sequence cannot be applied because of the wide variations in facial bone fractures. The decision on which sequence to use depends on the pattern of facial fractures with respect to the "immobile to mobile" and "simple to complicated" principles.

\section{REFERENCES}

1. Manson PN, Clark N, Robertson B, Crawley WA. Comprehensive management of pan-facial fractures. J Craniomaxillofac Trauma 1995;1:43-56.

2. Markowitz BL, Manson PN. Panfacial fractures: organization of treatment. Clin Plast Surg 1989;16:105-14.

3. Wenig BL. Management of panfacial fractures. Otolaryngol Clin North Am 1991;24:93-101

4. Degala S, Sundar SS, Mamata KS. A Comparative Prospective Study of Two Different Treatment Sequences i.e. Bottom Up-Inside Out and Topdown-Outside in, in the Treatment of Panfacial Fractures. J Maxillofac Oral Surg 2015;14:986-94.

5. de Melo WM, Sonoda CK, Shinohara EH, Garcia IR Jr. Using the "bottom-up and outside-in" sequence for panfacial fracture management: does it provide a clinical significance? J Craniofac Surg 2013;24:e479-81.

6. He D, Zhang Y, Ellis E, 3rd. Panfacial fractures: analysis of 33 cases treated late. J Oral Maxillofac Surg 2007;65:2459-65.

7. Curtis W, Horswell BB. Panfacial fractures: an approach to manage- ment. Oral Maxillofac Surg Clin North Am 2013;25:649-60.

8. Kim JH, Youn CY, Park ES, Tark MS, Lee YM. A clinical experience and treatment of panfacial fracture. J Korean Cleft Palate-Craniofac Assoc 2003;4:1-8.

9. Yang R, Zhang C, Liu Y, Li Z, Li Z. Why should we start from mandibular fractures in the treatment of panfacial fractures? J Oral Maxillofac Surg 2012;70:1386-92.

10. Manson PN, Clark N, Robertson B, Slezak S, Wheatly M, Vander Kolk $\mathrm{C}$, et al. Subunit principles in midface fractures: the importance of sagittal buttresses, soft-tissue reductions, and sequencing treatment of segmental fractures. Plast Reconstr Surg 1999;103:1287-306.

11. Gruss JS, Phillips JH. Complex facial trauma: the evolving role of rigid fixation and immediate bone graft reconstruction. Clin Plast Surg 1989;16:93-104.

12. Gruss JS, Mackinnon SE. Complex maxillary fractures: role of buttress reconstruction and immediate bone grafts. Plast Reconstr Surg 1986;78:9-22.

13. Tullio A, Sesenna E. Role of surgical reduction of condylar fractures in the management of panfacial fractures. Br J Oral Maxillofac Surg 2000;38:472-6

14. Pau M, Reinbacher KE, Feichtinger M, Navysany K, Karcher H. The mandibular symphysis as a starting point for the occlusal-level reconstruction of panfacial fractures with bicondylar fractures and interruption of the maxillary and mandibular arches: report of two cases. J Craniomaxillofac Surg 2014;42:e51-6.

15. Merville L. Multiple dislocations of the facial skeleton. J Maxillofac Surg 1974;2:187-200. 